\title{
EFFECT OF MAGNETICALLY TREATED WATER AND TRAINING METHODS ON SOME YIELD QUANTITATIVE TRAITS OF THREE GENOTYPES OF MUSKMELON (Cucumis melo L. ) GROWN UNDER GREENHOUSE CONDITIONS
}

(Received:16.6.2015)

\author{
By \\ A. M. A. Al-Shammary and R . H. Asmaael \\ Department of Horticulture \& Landscape, College of Agriculture, University of Diyala, Iraq
}

\begin{abstract}
The experiment was conducted at new Baquba Nursery, Diyala Agriculture Directorate, Iraq, during 2014 season. The objective of the study was to study the effect of magnetically treated water and training methods on yield quantitative traits of three genotypes of musk melon (Cucumis melo L.).

The experiment included 24 treatments as combinations of three genotypes of muskmelon RAND, NADA, and IDEAL, with two types of water (natural water, magnetically treated water 3000 Gauss), and using four training methods (without training, one stem, two stems, three stems). Factorial experiment with Spilt - Split plots system, in completely randomized complete Block Design CRBD with three replications were used. Duncan test as used to examine the significant differences among means. Result showed superiority of RAND genotype in fruit weight while IDEAL genotype had significant difference in fruit number per plant. Plants irrigated with magnetically treated water showed superiority in fruit number per plant, high yield per plant, and total high yield per green house. Plants trained on one stem showed least number of days required for first fruit maturity. Plants trained on two stems showed superiority in high yield per plant, and total high yield per green house. Plants trained on three stems significant differences in fruit number per plant. IDEAL genotype trained with three stems had a high number of fruit. Plants trained with three stems and irrigated with magnetically treated water showed high number of fruit. RAND genotype irrigated with natural water, and trained on three stems showed superiority in fruit weight. NADA genotype trained on two stems and irrigated with magnetically-treated water showed superiority in yield per plant, and total yield per green house. IDEAL genotype grown without training and irrigated with magnetically treated water showed least number of days required to fruit maturity. IDEAL genotype trained on three stems and irrigated with magnetically treated water showed superiority in fruit number.
\end{abstract}

Key words: musk melon, yield, Training, Magnetically treated water.
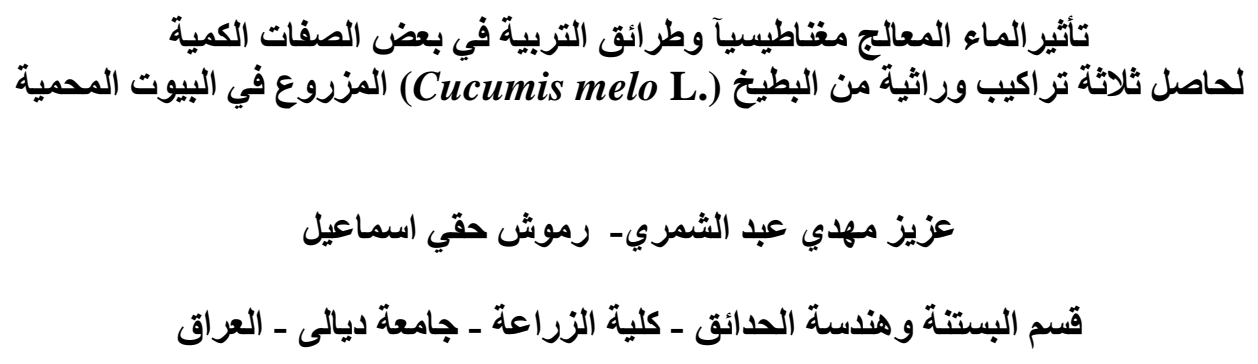

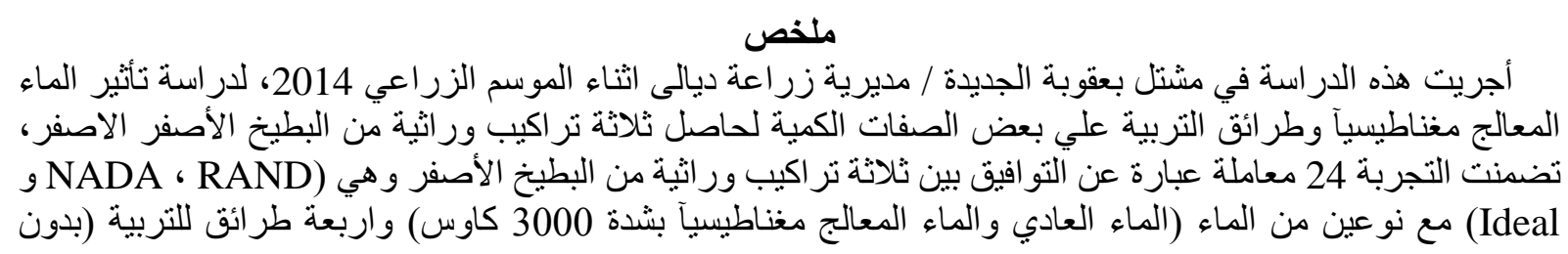


تقليم، ساق واحدة، ساقين، وثلاثة سيقان). نفذت تجربة عاملية وفق نظام القطع المنشقة ـ المنشقة S.S.P في تصميم R.C.B.D وبثلاث مكررات، أختبرت معنوية الفرق بين المتوسطات وفق اختية اختبار دنكن متعدد الحدود.

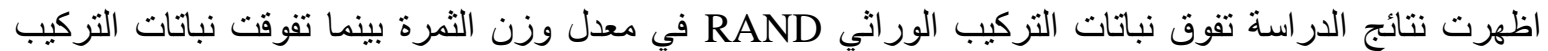

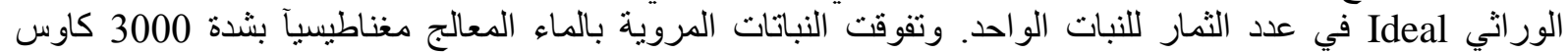

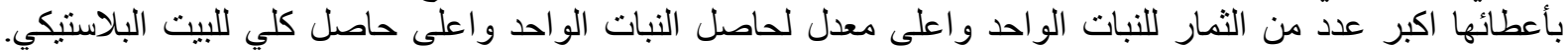

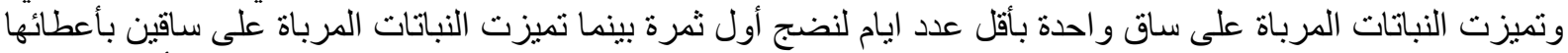

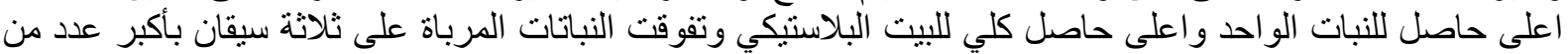

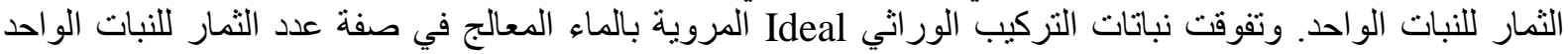

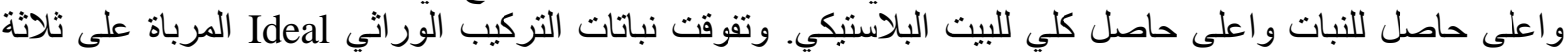

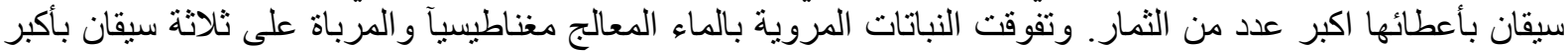

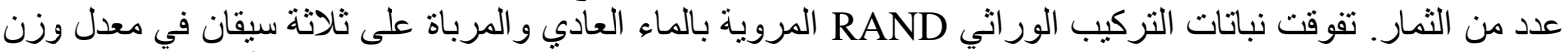

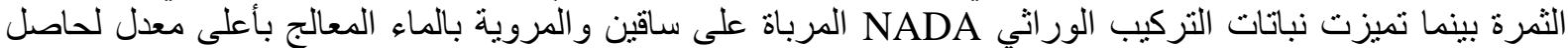

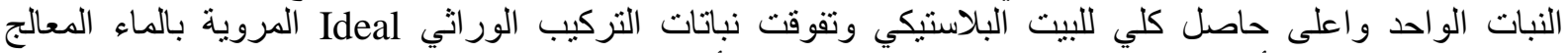

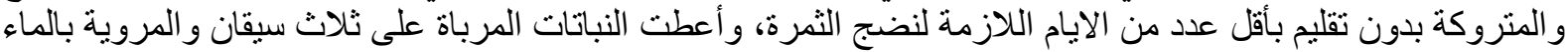

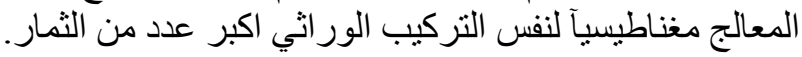
الكلمات المفتاحية : البطيخ ، الحاصل، طرّيقة التربية ، الماء المعاء المعالج مغناطيسيا

ان حاصل النبات يختلف بأختلاف التراكيب الوراثية،

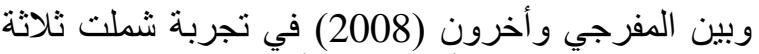
اصناف من البطيخ الأصفر الى أن هنات في فئك فروق معنوية

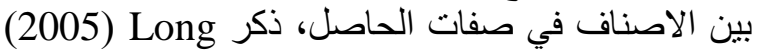

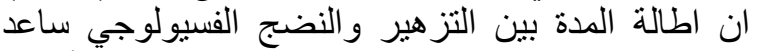

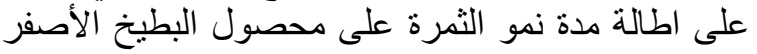

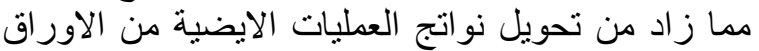

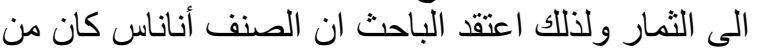
Shoemaker الاصناف المتأخرة في الحاصل. أوضان and William اصناف من البطيخ الأصفر بين فيها اختلاف الاصناف الإناف

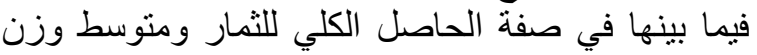

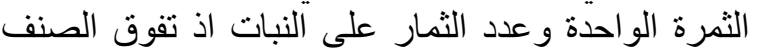
Starsweet الاصناف، وتفوق الصنف RML8726 في صفة منوسط

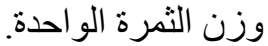

تؤثر الطاقة المغناطيسية على الماءة الماء من خلادل طبيعة

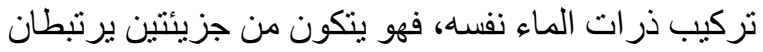

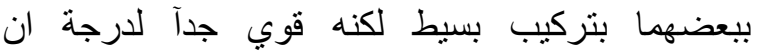

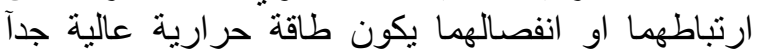

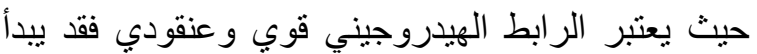

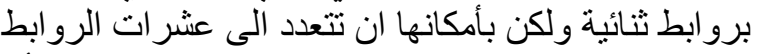

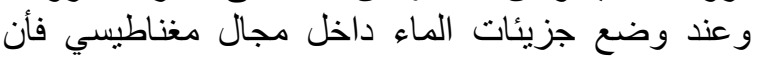

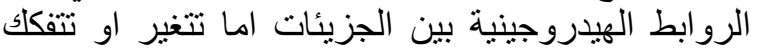

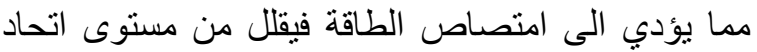
اجزاء الماء فيما بينها ويزيد من قابلية التحليل الكهربائي

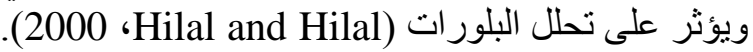

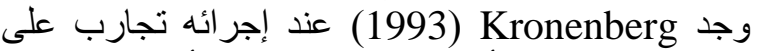
محاصيل مختلفة أن نباتات البطيخ الأصفر والهبارب القطن المروية بالماء المعالج أعطت زيادة في النئ النمو والحاصل

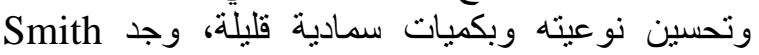
(2005) في ولاية كاليفورنيا ان الماء المعالج مغناطيسياً

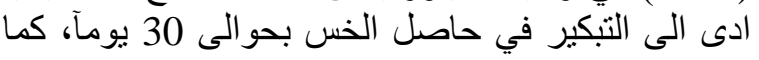

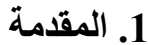

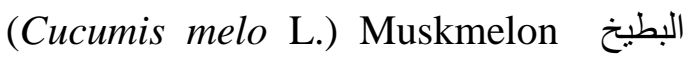

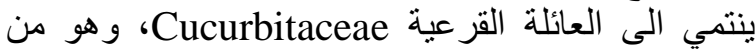
المحاصيل المهمة اقتصادياً في العالم اذ تجاوزت المئ المساحة

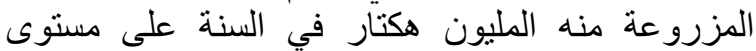

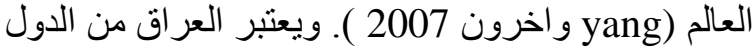

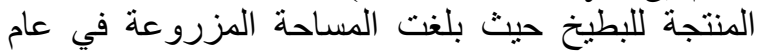

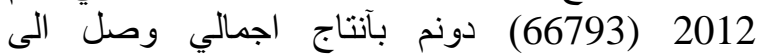

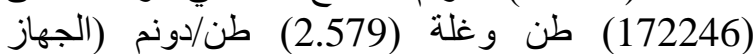

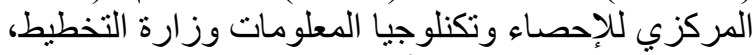

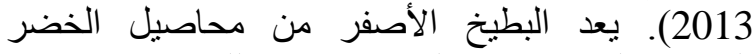

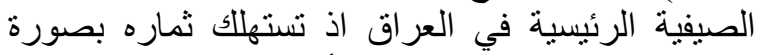

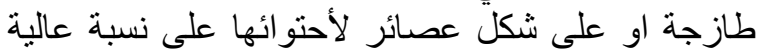

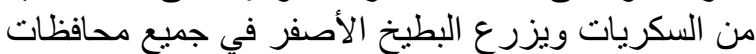

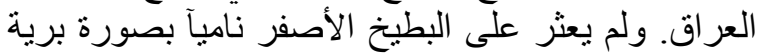

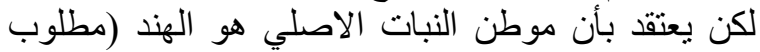
واخرون، 1989). تحصد ثمار البطيخ الأصفر مكتملة الأني

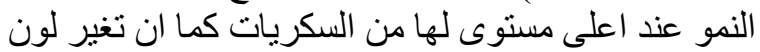

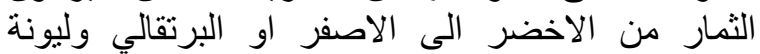

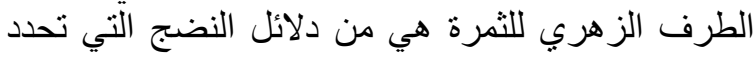
موعد الجني وطول مدة التخزين (مطلوب واخلخ الخرون، (1981

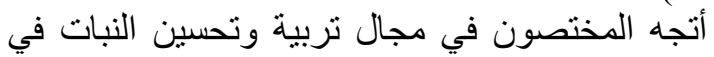

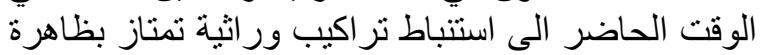
قوة الهجين في العديد من المحاصيل ولاطيل تلإيما محاصيل

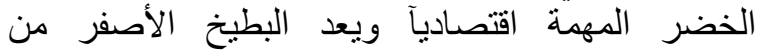

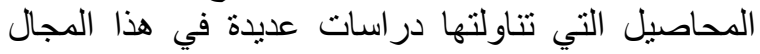

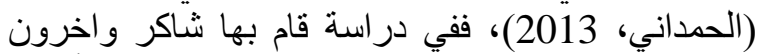
(2000) لانتخاب سلالات من اصناف البطيخ الأصفر

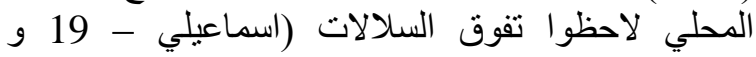

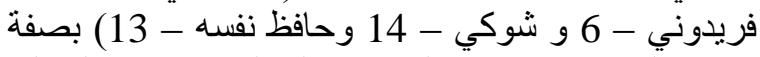

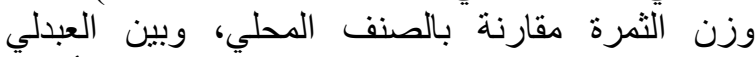
(2007) في در اسة لتحسين بعض صفات البطيخ الأصفر 


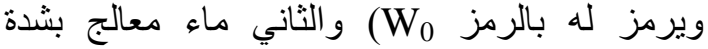

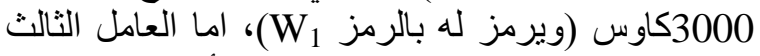

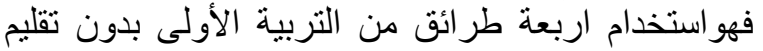

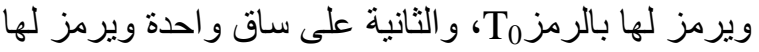

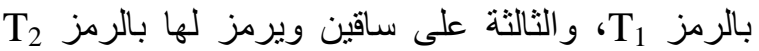

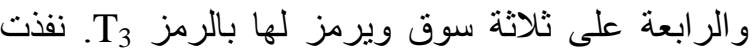

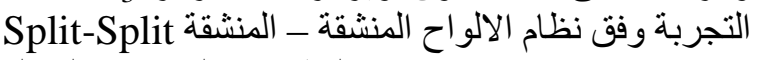
plot design

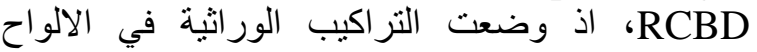

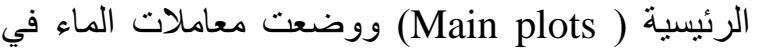

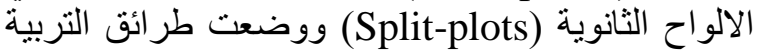
في الالو اح تحت الثنانوية.

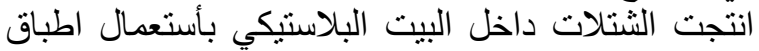

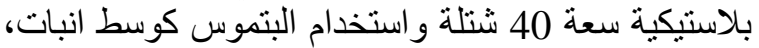

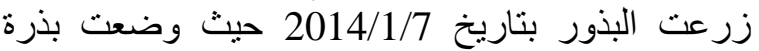

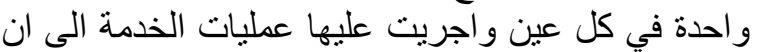

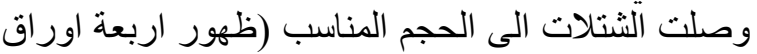

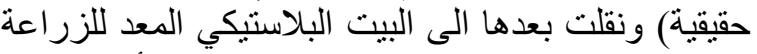

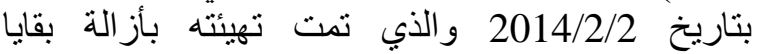
المحصول السابق ثم حر اثثة التربة لاكثر من مرة وتبن وتنعيمها

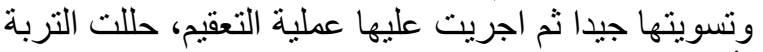

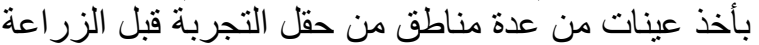

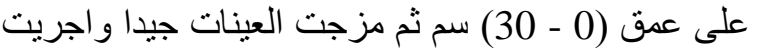

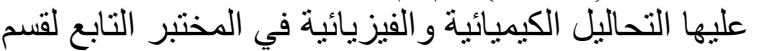
علوم التربة والموارد المائية في كلية الزرائية المة جامعة

زرعت الشتلات على مصاطب بعرض 80 سم لئل وبثمانية خطوط زر زاعة (خطين لكل مصطبة) وبمسافة الكبة

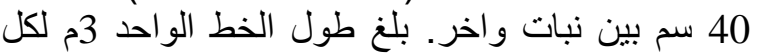

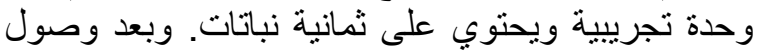

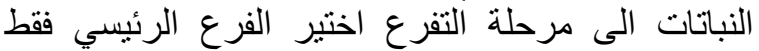

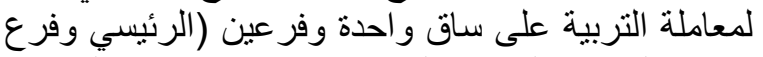
جانبي) لطريقة التربية على ساقين وثثلاثة فروع (الرين الرئيني

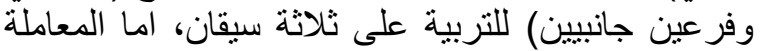
الر ابعة فتركت النباتات بدون تقليه تليه. اجريت عمليات التسليق وخدمة المحصول والتئن والري حسب الحاجة واخذت العينات من خمسة نباتات عشولة الئاً

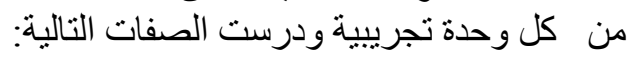

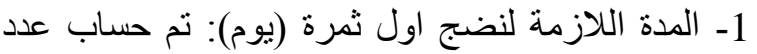
الايام التي استغرقتها النباتات لنضج النئ اول ثمرة فئن فيها ابتداء من الزر اعة في الحقل. 2- متوسط عدد الثمار للنبات الواحد (ثمرة/نبات):تم حساب عدد الثمار في الوحدة التجربية من بداية الجني التئي

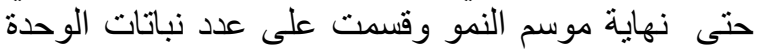
التجربية وفق المعادلة آلاتية:

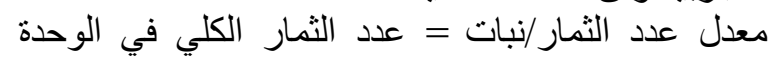
التجربية / عدد النباتات في الوحدة التجربية.

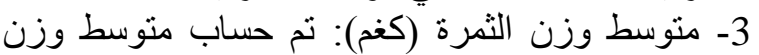

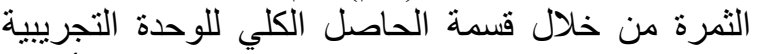
على عدد الثمار في الوحدة التجريبية وفق المعادلة الأتية :
حصل على زيادة في حاصل كل من الحنطة والذرة

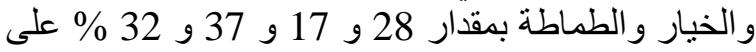
التو الي. اكد Basant و Grewal Gra (2009) ان معاملة مياه

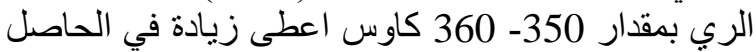

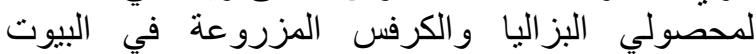

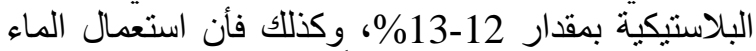

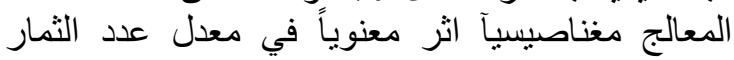
و الحاصل الكلي لمحصول الرقي ( المعاضيدي وآخرون،

تعد طريقة التربية من العوامل المؤثرة في كمية

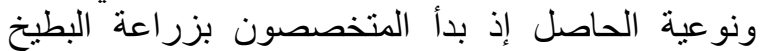
الأصفر في البيئة المحمية إلى تربية النية النباتات رأسياء و المقصود بطريقة التربية هنا هو البناء الهندسي للنبة النباتات

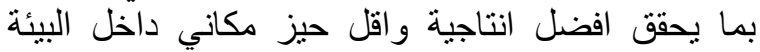
المحمية بما لا يعيق عمليات الخدمة ويقلل من الإني الاضر الترار

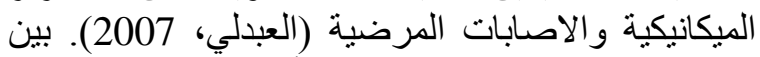

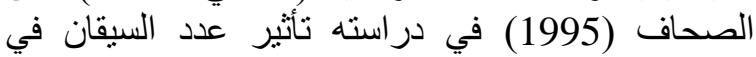

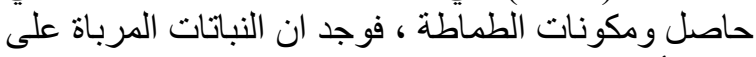

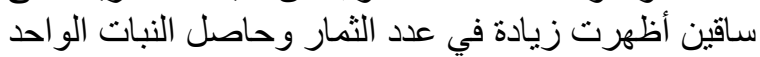

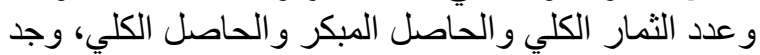

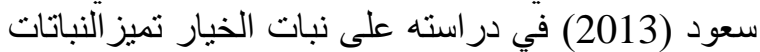
المرباة على ساقين في حاصل فلى النبات الواحد التد ومعدل حاصل النباتات في المتر المربع وفي عدد الثيات الثمار للنبات

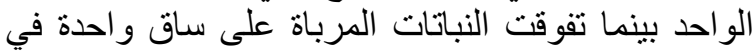

صفة معدل طول الثمرة وقطر الثرات الثرة، اوضى الثراف

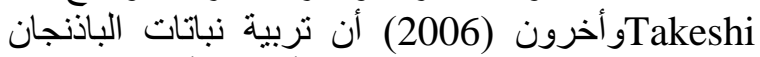

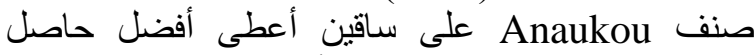

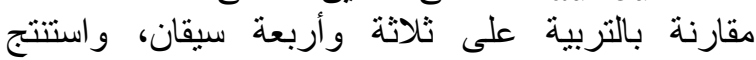

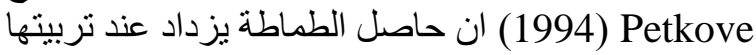

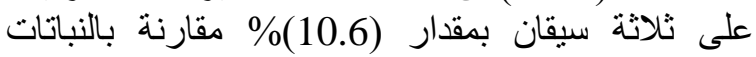
المرباة على ساقين. ان الهدف من هذه الدراسة هو اختيار افضل نركيب الإني

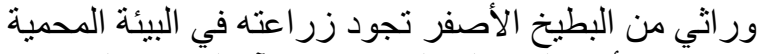

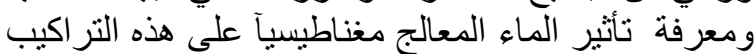

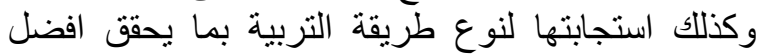

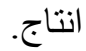

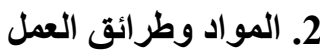

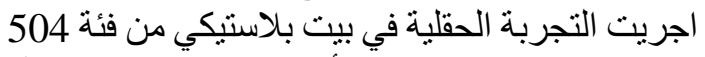

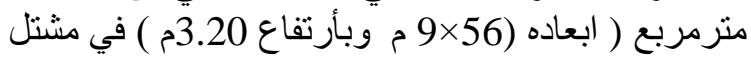

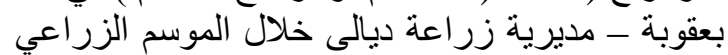
.2014

تضمنت الدراسة ثناثة عوامل الاول زراعة ثناثة تر اكيب ور اثية (هجن جديدة) من البطيخ الأصفر المدخلة الأنة

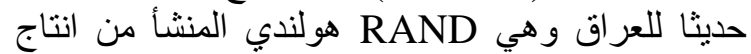

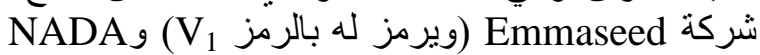

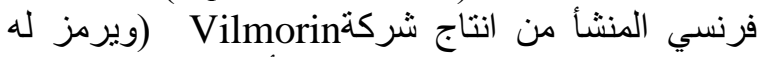

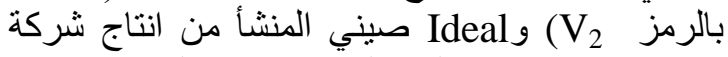
Syngenta نوعين من الماء الاول ماء عادي (غير معالج مغناطيسياً 


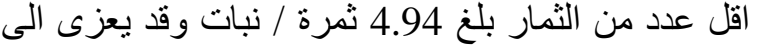

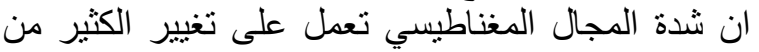

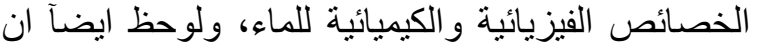

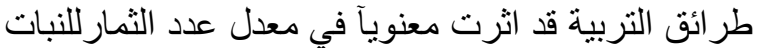

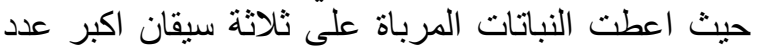

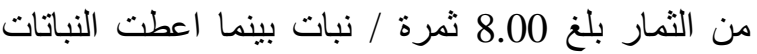

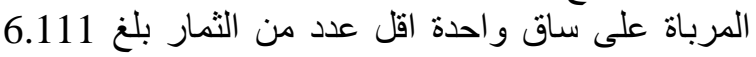

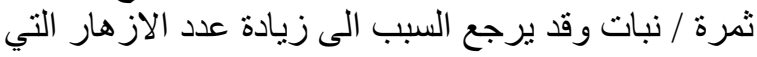

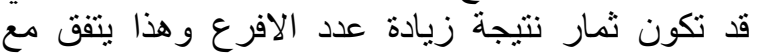
ماوجده التحافي وأخرون (2011) في در استه على نبات التهات

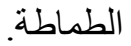

يبين الجدول (2) وجود فروق معنوية بالنسبة

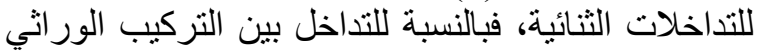

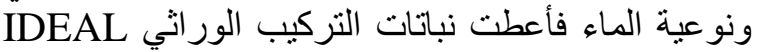

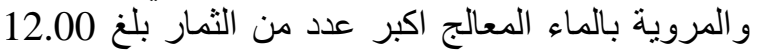

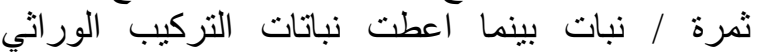

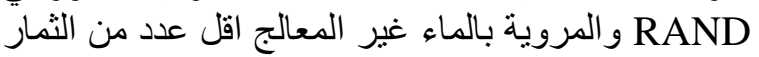

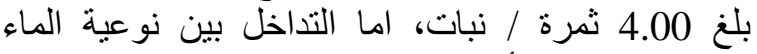

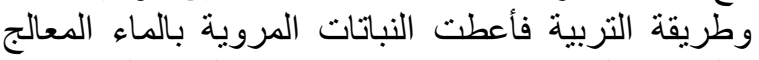
و المرباة على ثلاث سيقان اكبر عدد من الثنات الثمار بلغ 10.22

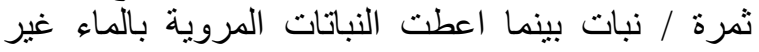

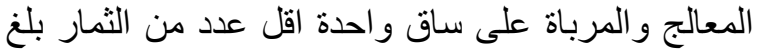

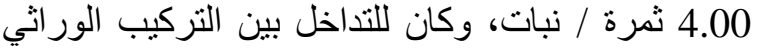

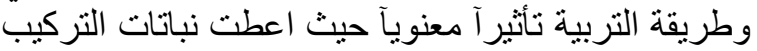

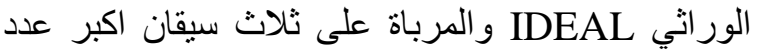

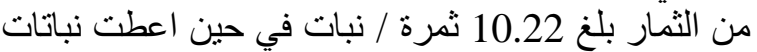

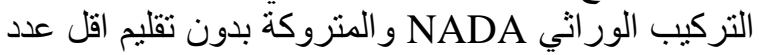

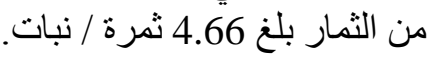

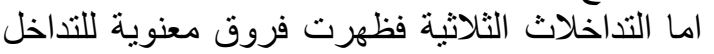
بين التر اكيب الور اثية ونوعية الماء وطر ائق التربية حيث فيث

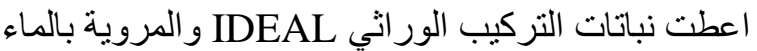

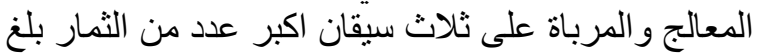

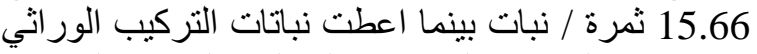

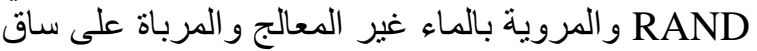

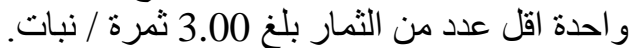

3.3. متوسط وزن الثمار(كفم) الثمار بلغ

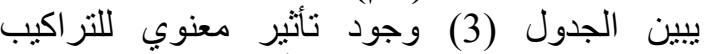

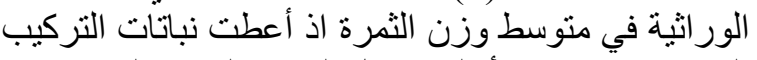

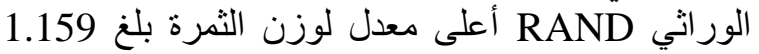

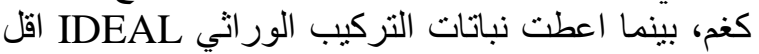

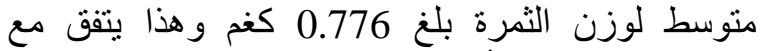

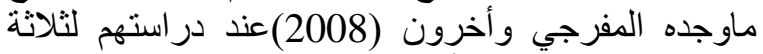
اصناف من البطيخ الأصفر في الترب الجنئ الجبسية. وبينت النتائج عدم وجود تأثير معنوي بالنسبة لنو عية الماء وكذللك طر ائق التربية. - مبائ. اما بالنسبة للتداخلات الثنائية يتضح عدم وجود تأثير

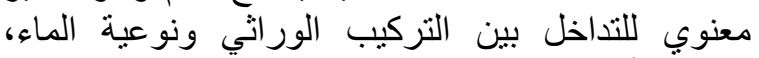

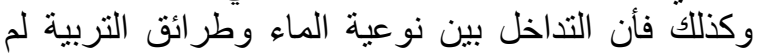

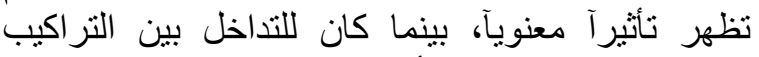

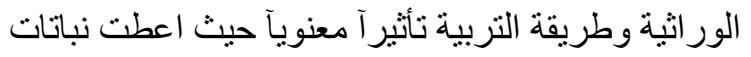

متوسط وزن الثمرة (كغم) = حاصل الوحدة التجربية

(كغم) /عدد ثمار نباتات الوحدة التجربية نفسها.

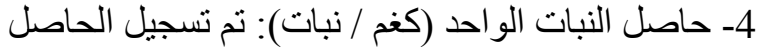

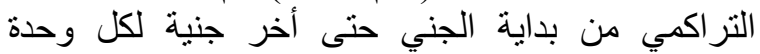

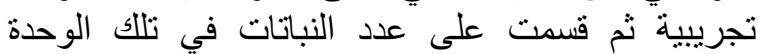
التجريبية.

5- الحاصل الكلي للبيت البلاستيكي (طن / البيت البيت البلاستنيكي):وتم حسابه وفق الباهي المعادلة التالّية: حاصل البيت البلاستيكي = حاصل البن النبات الواحد (كغم) × عدد النباتات في البيت البلاستيكي / 1000. تم تحليل النتائج احصائيا وحسب النيب التصميم المستخدم

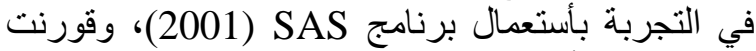
المتوسطات بأختبار دنكن متعدد الحدود (الراوي وخلف

الله، 2000).

\section{3. النتائج والمناقشة}

1.3.المدة اللازمة لنضج اول ثمرة (يوم)

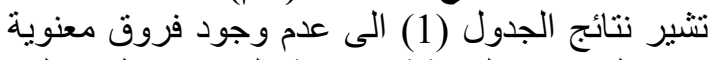

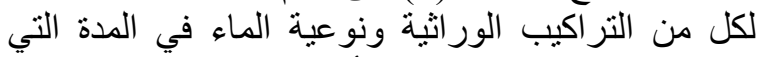

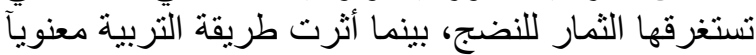
على هذه الصفة حيث استغرقت النباتات المرباة على لاتى ساق

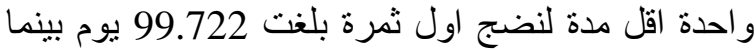

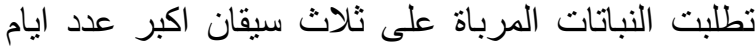

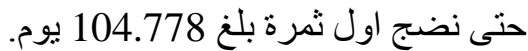

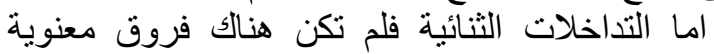
للتداخل بين التراكيب الور اثية ونوعية التية الماء وكذللك بين

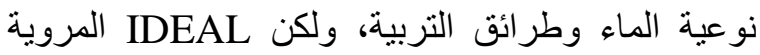
اظهر التداخل بين التزراكيب الوراثية وطرنة ولثرائق التربية

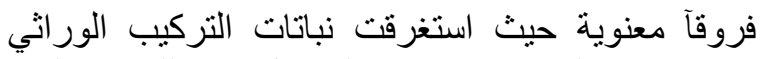
IDEAL

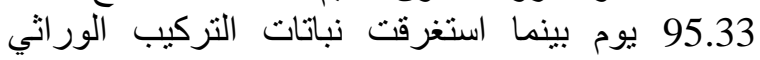
NADA و المرباة على ثلاث سوق اكبر عدد من الايام لنضج اول ثمرة بلغ المرباة على 110.33 يوم.

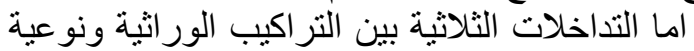

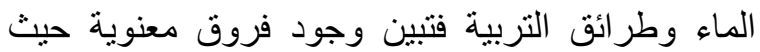

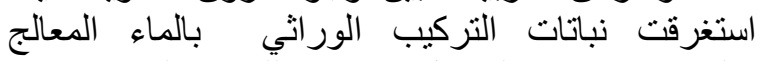

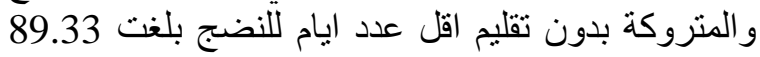

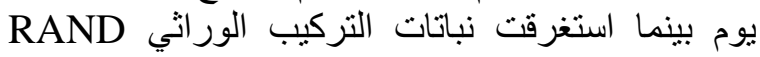

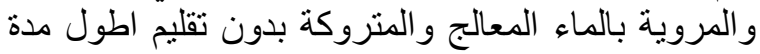

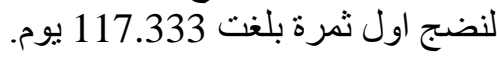

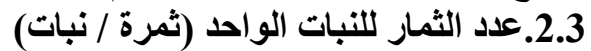

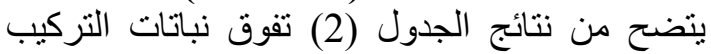
الور اثي IDEAL معنوياً في عدد ثمار ها حيث بلغ نلغ

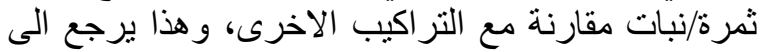

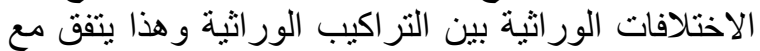

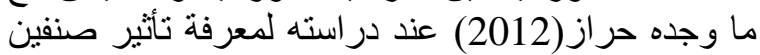

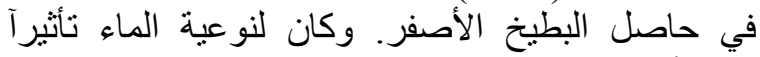
معنوياً في معدل عدد الثمار للنبات حيث الثيث انتجت النبات النباتات

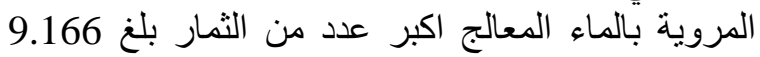
ثمرة/نبات واعطت النباء المعاج النبر المروية بالماء غير المعالج 
جدول (1): تأثير التركيب الوراثي والماء المعالج مغناطيسياّ وطرائق التربية وتداخلاتها في المدة اللازمة لنضج

\begin{tabular}{|c|c|c|c|c|c|c|}
\hline \multirow{3}{*}{$\begin{array}{l}\text { التداخل } \\
\mathbf{V} \times \mathbf{w}\end{array}$} & & & & & \multicolumn{2}{|c|}{ اول ثمرة (يوم)*. } \\
\hline & \multicolumn{4}{|c|}{ طرائق التربية } & \multirow{2}{*}{ نوعية الماء } & \multirow{2}{*}{ التوراثيبة } \\
\hline & $\mathbf{T}_{3}$ & \begin{tabular}{l|l}
$\mathbf{T}_{2}$ &
\end{tabular} & $\mathbf{T}_{1}$ & $\mathbf{T}_{0}$ & & \\
\hline $\begin{array}{c}102.00 \\
a\end{array}$ & $\begin{array}{c}104.00 \\
\text { bc }\end{array}$ & $\begin{array}{c}103.333 \\
\text { bc }\end{array}$ & $\begin{array}{c}102.00 \\
\text { bed }\end{array}$ & $\begin{array}{c}98.667 \\
\text { cd }\end{array}$ & $\mathbf{W}_{\mathbf{0}}$ & \multirow[b]{2}{*}{$\mathbf{V}_{1}$} \\
\hline $\begin{array}{c}106.083 \\
\text { a }\end{array}$ & $\begin{array}{c}106.667 \\
\text { abc }\end{array}$ & $\begin{array}{c}98.333 \\
\text { cd }\end{array}$ & $\begin{array}{c}102.00 \\
\text { bed }\end{array}$ & $\begin{array}{c}117.33 \\
\mathrm{a}\end{array}$ & $\mathbf{W}_{1}$ & \\
\hline $\begin{array}{c}105.583 \\
\text { a }\end{array}$ & $\begin{array}{c}114.00 \\
\text { ab }\end{array}$ & $\begin{array}{c}101.00 \\
\text { bed }\end{array}$ & $\begin{array}{c}100.00 \\
\text { cd }\end{array}$ & $\begin{array}{c}107.33 \\
\text { abc }\end{array}$ & $\mathbf{W}_{\mathbf{0}}$ & \multirow[b]{2}{*}{$\mathbf{V}_{2}$} \\
\hline $\begin{array}{c}101.583 \\
a\end{array}$ & $\begin{array}{c}106.667 \\
\text { abc }\end{array}$ & $\begin{array}{c}99.333 \\
\text { cd }\end{array}$ & $\begin{array}{c}99.667 \\
\text { cd }\end{array}$ & $\begin{array}{c}100.66 \\
\text { cd }\end{array}$ & $\mathbf{W}_{1}$ & \\
\hline $\begin{array}{c}99.333 \\
\mathbf{a}\end{array}$ & $\begin{array}{c}\mathbf{9 8 . 3 3 3} \\
\text { cd }\end{array}$ & $\begin{array}{c}100.00 \\
\text { cd }\end{array}$ & $\begin{array}{c}97.667 \\
\text { cd }\end{array}$ & $\begin{array}{c}101.33 \\
\text { bed }\end{array}$ & $\mathbf{W}_{\mathbf{0}}$ & \multirow{2}{*}{$\mathbf{V}_{3}$} \\
\hline $\begin{array}{c}96.083 \\
\text { a }\end{array}$ & $\begin{array}{c}99.00 \\
\text { cd }\end{array}$ & $\begin{array}{l}99.00 \\
\text { cd }\end{array}$ & $\begin{array}{c}97.00 \\
\text { cd }\end{array}$ & $\begin{array}{c}89.33 \\
\text { d }\end{array}$ & $\mathbf{W}_{1}$ & \\
\hline & $\begin{array}{c}104.778 \\
\mathrm{~A}\end{array}$ & $\begin{array}{c}100.167 \\
\text { AB }\end{array}$ & $\begin{array}{c}99.722 \\
\text { B }\end{array}$ & $\begin{array}{c}102.44 \\
\text { AB }\end{array}$ & \multicolumn{2}{|c|}{ متوسطات طرائق التربية } \\
\hline \multicolumn{7}{|c|}{ التذاخل الثنائي بين التراكيب الوراثية وطرائق التربية } \\
\hline متوسطات & \multicolumn{4}{|c|}{ طر ائق التربية } & \multirow{2}{*}{\multicolumn{2}{|c|}{ التراكيب الوراثية }} \\
\hline التراكيب الور اثية & T3 & T2 & T1 & T0 & & \\
\hline $\begin{array}{c}104.042 \\
\text { A }\end{array}$ & $\begin{array}{c}105.333 \\
\text { Abc }\end{array}$ & $\begin{array}{c}100.833 \\
\text { bcd }\end{array}$ & $\begin{array}{c}102.00 \\
a-d\end{array}$ & $\begin{array}{c}108.00 \\
\text { ab }\end{array}$ & \multicolumn{2}{|c|}{$\mathbf{V}_{1}$} \\
\hline $\begin{array}{c}103.583 \\
A\end{array}$ & $\begin{array}{c}110.333 \\
\mathrm{a}\end{array}$ & $\begin{array}{c}100.167 \\
\text { bed }\end{array}$ & $\begin{array}{c}99.833 \\
\text { bcd }\end{array}$ & $\begin{array}{c}104.00 \\
a-d\end{array}$ & \multicolumn{2}{|c|}{$\mathbf{V}_{2}$} \\
\hline $\begin{array}{c}97.708 \\
\text { A }\end{array}$ & $\begin{array}{c}98.667 \\
\text { cd }\end{array}$ & $\begin{array}{c}99.500 \\
\text { bed }\end{array}$ & $\begin{array}{c}97.333 \\
\text { cd }\end{array}$ & $\begin{array}{c}95.333 \\
d\end{array}$ & \multicolumn{2}{|c|}{$\mathbf{V}_{3}$} \\
\hline \multicolumn{7}{|c|}{ التداخل الثنائي بين نوعية الماء وطرائق التربية } \\
\hline متوسطات نوعية & \multicolumn{4}{|c|}{ طر ائق التربية } & \multirow{2}{*}{\multicolumn{2}{|c|}{ نوعية الماء }} \\
\hline الماء & $\mathbf{T}_{3}$ & $\mathbf{T}_{2}$ & $\mathbf{T}_{1}$ & $\mathbf{T}_{0}$ & & \\
\hline $\begin{array}{c}102.306 \\
\mathrm{~A}\end{array}$ & $\begin{array}{c}105.444 \\
\mathrm{a}\end{array}$ & $\begin{array}{c}101.444 \\
\text { a }\end{array}$ & $\begin{array}{c}99.889 \\
\mathrm{a}\end{array}$ & $\begin{array}{c}102.44 \\
\text { a }\end{array}$ & \multicolumn{2}{|c|}{$\mathbf{W}_{\mathbf{0}}$} \\
\hline $\begin{array}{c}101.250 \\
\mathrm{~A}\end{array}$ & $\begin{array}{c}104.111 \\
\text { a }\end{array}$ & $\begin{array}{c}98.889 \\
\text { a }\end{array}$ & $\begin{array}{c}99.556 \\
\mathrm{a}\end{array}$ & $\begin{array}{c}102.44 \\
\text { a }\end{array}$ & \multicolumn{2}{|c|}{$\mathbf{W}_{1}$} \\
\hline
\end{tabular}

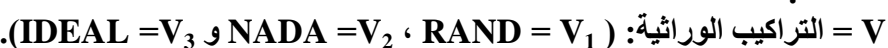

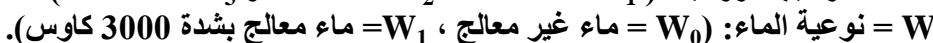

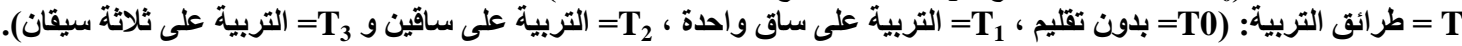


جدول (2) :تأثير التركيب الوراثي والماء المعالج مغناطيسياً وطرائق التربية وتداخلاتها في متوسط عدد الثمار للنبات الواحل (ثمرة / نبات)*

\begin{tabular}{|c|c|c|c|c|c|c|}
\hline \multirow{2}{*}{$\begin{array}{l}\text { التداخل } \\
\mathbf{V} \times \mathbf{w}\end{array}$} & \multicolumn{4}{|c|}{ طرائق التربية } & \multirow{2}{*}{ نوعية الماء } & \multirow{2}{*}{ التراثية } \\
\hline & $\mathbf{T}_{3}$ & \begin{tabular}{l|l}
$\mathbf{T}_{2}$ \\
\end{tabular} & $\mathbf{T}_{1}$ & $\mathbf{T}_{\mathbf{0}}$ & & \\
\hline $\begin{array}{c}4.00 \\
d\end{array}$ & $\begin{array}{l}4.667 \\
\text { e-h }\end{array}$ & $\begin{array}{l}5.000 \\
\text { e-h }\end{array}$ & $\begin{array}{c}3.000 \\
\text { h }\end{array}$ & $\begin{array}{c}3.333 \\
\text { gh }\end{array}$ & $\mathbf{W}_{\mathbf{0}}$ & \multirow[b]{2}{*}{$\mathbf{V}_{1}$} \\
\hline $\begin{array}{c}7.750 \\
\text { b }\end{array}$ & $\begin{array}{c}7.333 \\
d-g\end{array}$ & $\begin{array}{c}5.667 \\
\text { d-h }\end{array}$ & $\begin{array}{c}9.000 \\
\text { cd }\end{array}$ & $\begin{array}{l}9.000 \\
\text { cd }\end{array}$ & $\mathbf{W}_{1}$ & \\
\hline $\begin{array}{c}4.250 \\
\text { cd }\end{array}$ & $\begin{array}{c}\text { 6.000 } \\
\text { d-h }\end{array}$ & $\begin{array}{c}4.000 \\
\text { fgh }\end{array}$ & $\begin{array}{c}3.333 \\
\text { gh }\end{array}$ & $\begin{array}{c}3.667 \\
\text { gh }\end{array}$ & $\mathbf{W}_{\mathbf{0}}$ & \multirow[b]{2}{*}{$\mathbf{V}_{2}$} \\
\hline $\begin{array}{c}7,750 \\
\text { b }\end{array}$ & $\begin{array}{c}7.667 \\
\text { c-f }\end{array}$ & $\begin{array}{c}11.333 \\
\text { bc }\end{array}$ & $\begin{array}{c}6.333 \\
d-h\end{array}$ & $\begin{array}{c}5.667 \\
\text { d-h }\end{array}$ & $\mathbf{W}_{1}$ & \\
\hline $\begin{array}{c}6.583 \\
\text { bc }\end{array}$ & $\begin{array}{c}6.667 \\
d-h\end{array}$ & $\begin{array}{c}8.333 \\
\text { cde }\end{array}$ & $\begin{array}{c}5.667 \\
\text { d-h }\end{array}$ & $\begin{array}{c}5.667 \\
\text { d-h }\end{array}$ & $\mathbf{W}_{\mathbf{0}}$ & \multirow{2}{*}{$\mathbf{V}_{3}$} \\
\hline \multirow[t]{2}{*}{$\begin{array}{c}12.00 \\
\text { a } \\
\end{array}$} & $\begin{array}{c}15.667 \\
\mathrm{a}\end{array}$ & $\begin{array}{c}13.333 \\
\text { ab }\end{array}$ & $\begin{array}{c}9.333 \\
\text { cd }\end{array}$ & $\begin{array}{c}9.667 \\
\text { cd }\end{array}$ & $\mathbf{W}_{1}$ & \\
\hline & $\begin{array}{c}\mathbf{8 . 0 0} \\
\mathbf{A}\end{array}$ & $\begin{array}{c}7.944 \\
\text { A }\end{array}$ & $\begin{array}{c}6.111 \\
\text { B }\end{array}$ & $\begin{array}{c}6.166 \\
\text { B }\end{array}$ & \multicolumn{2}{|c|}{ متوسطات طرائق التربية } \\
\hline \multicolumn{7}{|c|}{ التداخل الثنائي بين التراكيب الوراثية وطرائق التربية } \\
\hline \multirow{2}{*}{ التر اكيب الوراثية } & \multicolumn{4}{|c|}{ طرائق التربية } & \multirow{2}{*}{\multicolumn{2}{|c|}{ التراكيب الوراثية }} \\
\hline & $\mathbf{T}_{\mathbf{3}}$ & $\mathbf{T}_{2}$ & $\mathbf{T}_{1}$ & $\mathbf{T}_{\mathbf{0}}$ & & \\
\hline $\begin{array}{c}5.875 \\
\text { B }\end{array}$ & $\begin{array}{l}6.00 \\
\text { bed }\end{array}$ & $\begin{array}{c}5.333 \\
\text { bcd }\end{array}$ & $\begin{array}{l}6.00 \\
\text { bed }\end{array}$ & $\begin{array}{c}6.167 \\
\text { bed }\end{array}$ & \multicolumn{2}{|c|}{$\mathbf{V}_{1}$} \\
\hline $\begin{array}{c}6.00 \\
\text { B }\end{array}$ & $\begin{array}{c}6.833 \\
\text { bcd }\end{array}$ & $\begin{array}{c}7.667 \\
\text { b }\end{array}$ & $\begin{array}{c}4.833 \\
\text { cd }\end{array}$ & $\begin{array}{c}4.667 \\
d\end{array}$ & \multicolumn{2}{|c|}{$\mathbf{V}_{2}$} \\
\hline $\begin{array}{c}9.291 \\
\mathrm{~A}\end{array}$ & $\begin{array}{c}11.167 \\
a\end{array}$ & $\begin{array}{c}10.833 \\
\mathrm{a}\end{array}$ & $\begin{array}{c}7.500 \\
\text { bc }\end{array}$ & $\begin{array}{c}7.667 \\
\text { b }\end{array}$ & \multicolumn{2}{|c|}{$\mathbf{V}_{3}$} \\
\hline \multicolumn{7}{|c|}{ التداخل الثنائي بين نوعية الماء وطرائق التربية } \\
\hline 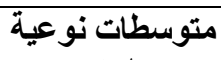 & \multicolumn{4}{|c|}{ طرائق التربية } & \multirow{2}{*}{\multicolumn{2}{|c|}{ 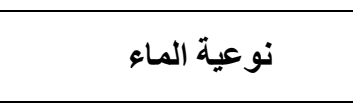 }} \\
\hline الماءع & $\mathbf{T}_{3}$ & $\mathbf{T}_{2}$ & $\mathbf{T}_{1}$ & $\mathbf{T}_{\mathbf{0}}$ & & \\
\hline $\begin{array}{c}4.944 \\
\text { B }\end{array}$ & $\begin{array}{c}5.777 \\
\text { c }\end{array}$ & $\begin{array}{c}5.777 \\
\text { c }\end{array}$ & $\begin{array}{l}4.000 \\
\text { c }\end{array}$ & $\begin{array}{c}4.222 \\
c\end{array}$ & \multicolumn{2}{|c|}{$\mathbf{W}_{\mathbf{0}}$} \\
\hline $\begin{array}{c}9.166 \\
\text { A }\end{array}$ & $\begin{array}{c}10.222 \\
\text { a }\end{array}$ & $\begin{array}{c}10.111 \\
\text { ab }\end{array}$ & $\begin{array}{c}8.222 \\
\text { ab }\end{array}$ & $\begin{array}{c}8.111 \\
b\end{array}$ & \multicolumn{2}{|c|}{$\mathbf{W}_{1}$} \\
\hline
\end{tabular}

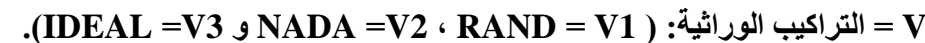

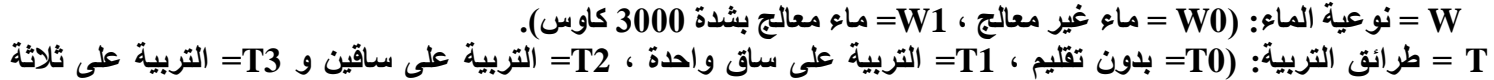


جلول (3) :تأثير التركيب الوراثي والماء المعالج مغناطيسياً وطر ائق التربية وتداخلاتها في متوسط وزن الثمار

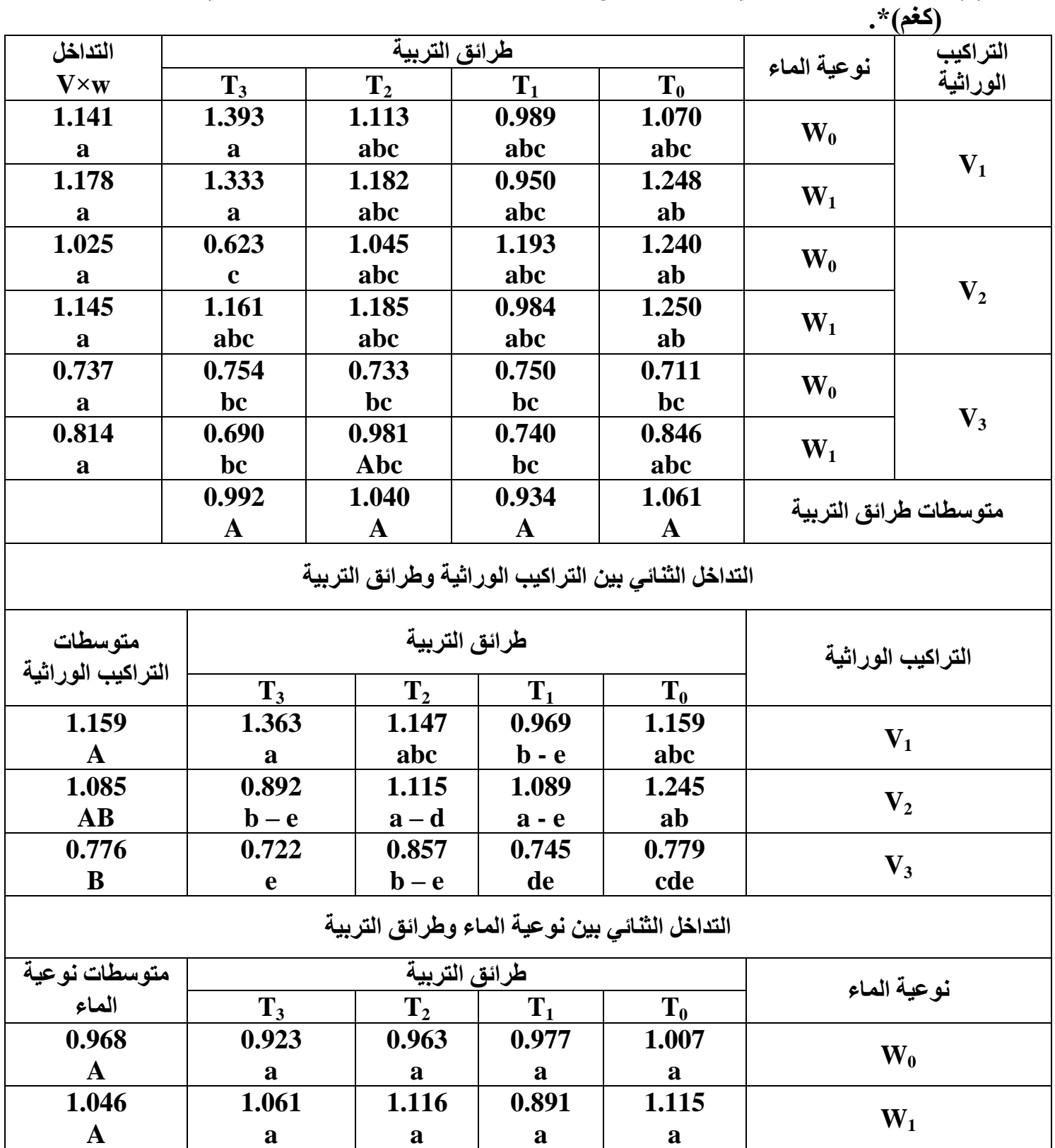


5.3.

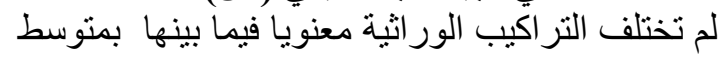

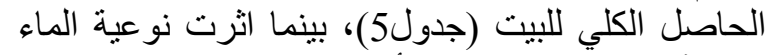

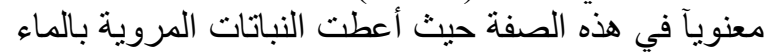

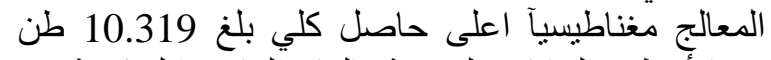

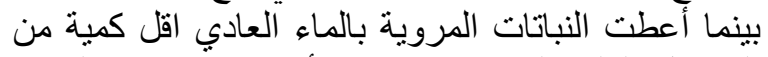

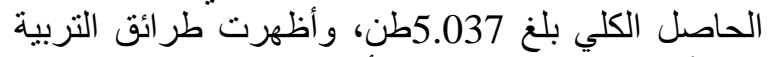

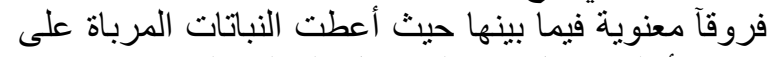

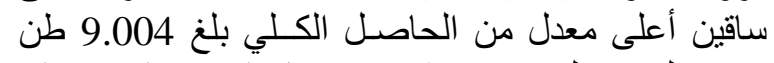

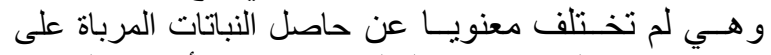

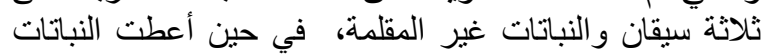

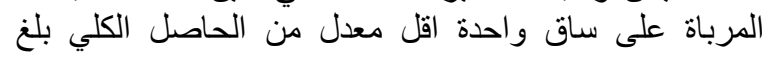
5.997

تظهر نتائج الجدول (5) التداخلات الثنائية، حيث لوحظ التركي

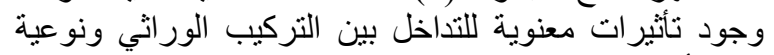

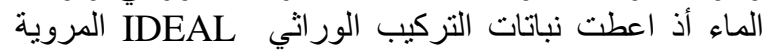

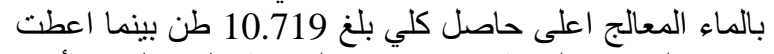

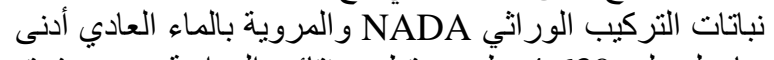

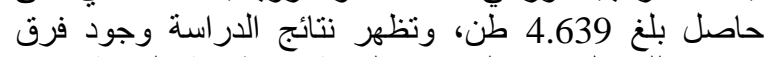
معنوي للتداخل بين التركيب الوراثي وطريقة التربية حيث التباني

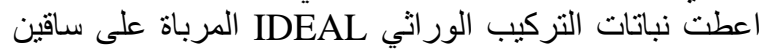
اعلى معدل بلغ 10.552 طن بينمات اعلى اعت نبات نباتات التركيب

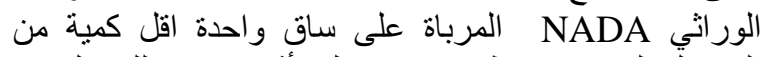

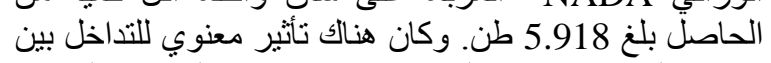

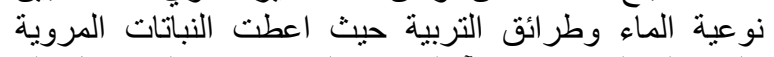

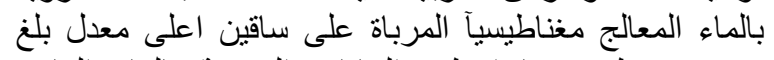

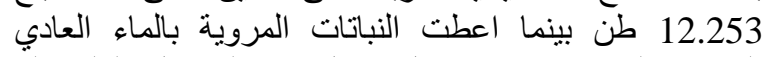

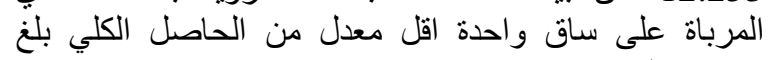
( 4.201

وكان للتداخل الثلاثي بين التر اكيب الور اثية ونو عية الماء

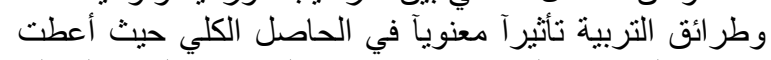

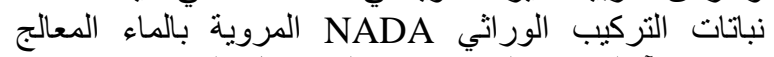

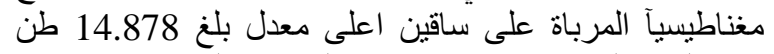

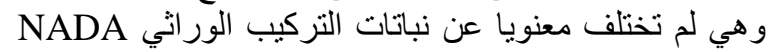

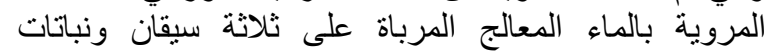

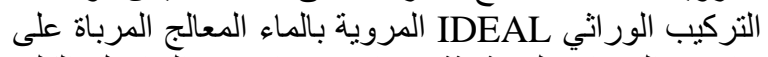

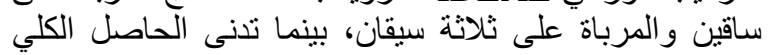

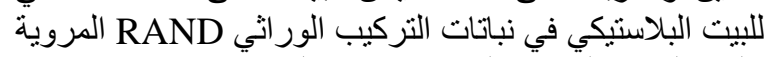

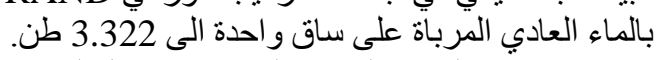

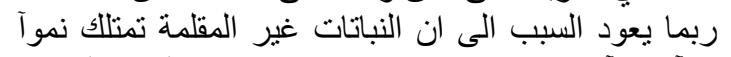

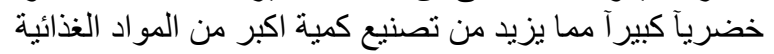

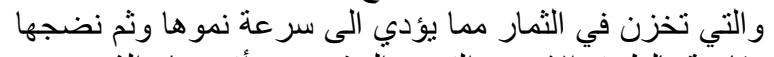

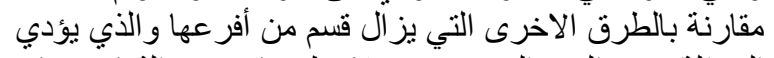

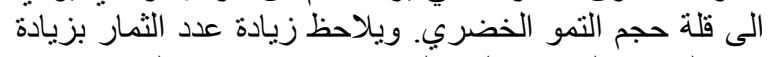

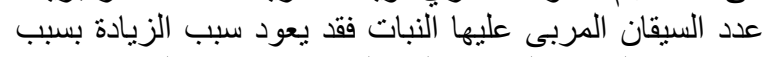

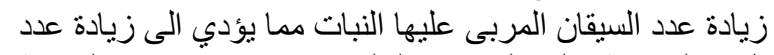

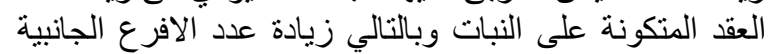

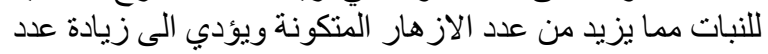

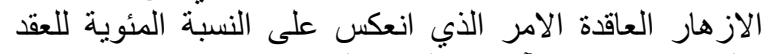

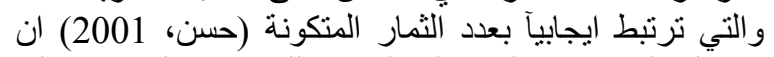

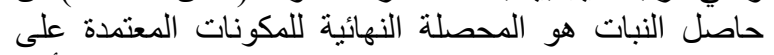

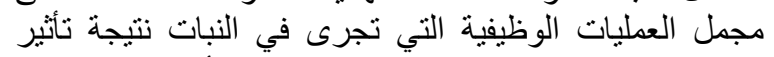

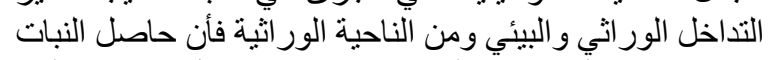

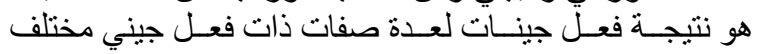

التركيب الوراثي RAND و المرباة على ثناثة سيقان اعلى معدل لوزن التئ الثمرة بلغ 1.36 كغم بينما أعطت نباتات التركيب الور اثي IDEAL والثرة بلغ المرباة على ثناث سيقان اقل معدل لوزن التُبرة التب بلغ 0.722 كغر.

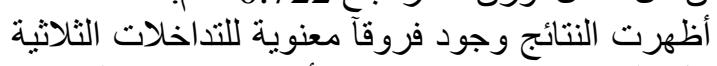

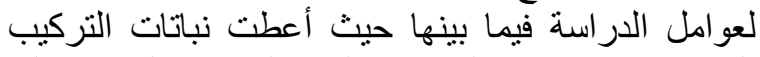

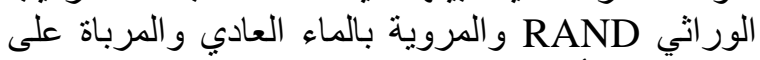

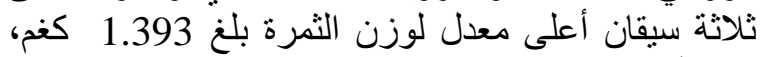

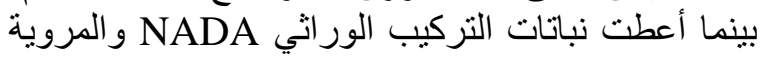

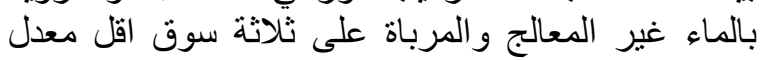
لوزن الثمرة (0.623 كغئ كنم).

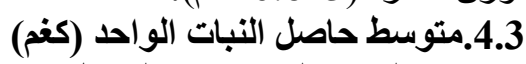
تبين النتائج الددونة في الجدات الواحدول (4) عدم) عدم وجود تأثير

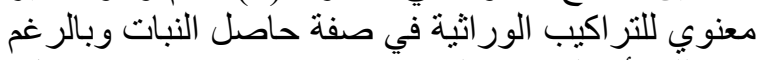

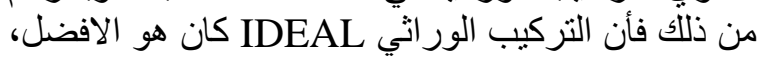

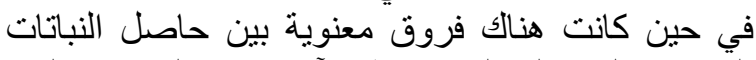

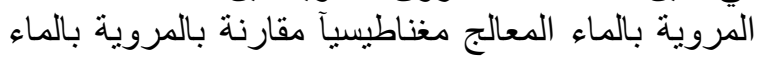

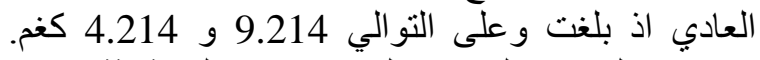
وتميزت النباتات المرباة على سلى ساقين و على ثلاثنة سيقان

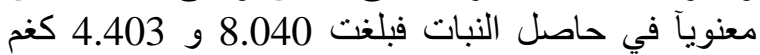

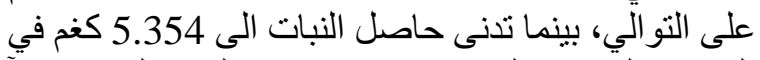

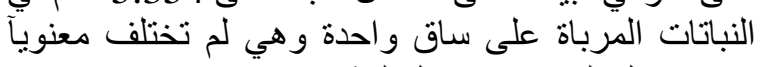
عن حاصل النباتات غير المقلمة.

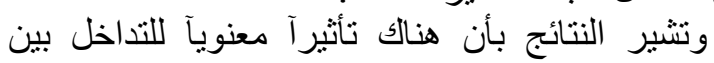

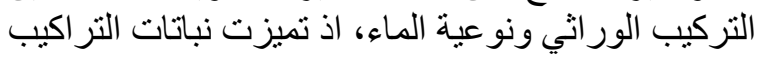

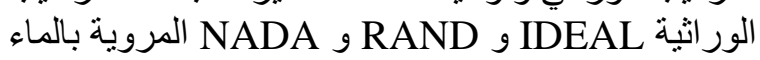
المعالج بأفضل حاصل للنبات بلغ و على الترتيب 8.898 و 9.570 و و

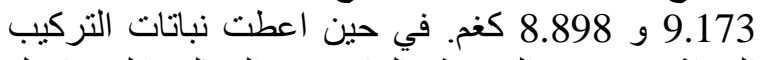

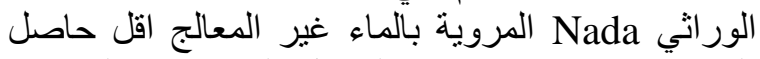
بلغ 4.142 كغ. كما الثر التداخل الثراء الثنائي بين الترائ التراكيب

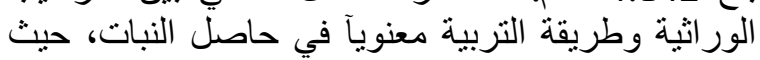

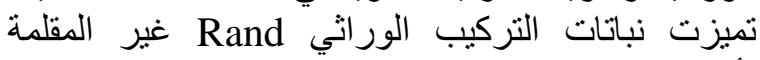

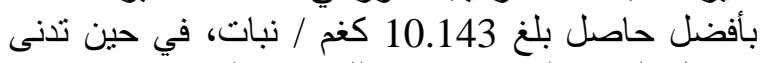

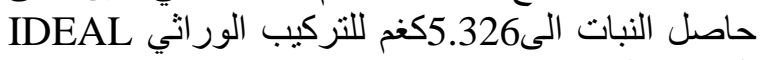
المربى على ساق و احدة. وتوضح النتائج ان للتداخل الثنائي بين نوعية الماء الثياء

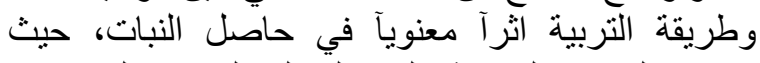

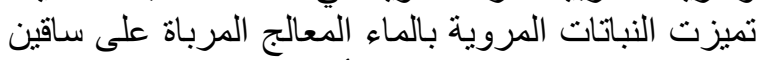
وثلاثة سوق و غير المقلمة بأفضل حاصل المبل بلغ وعلى وعلى

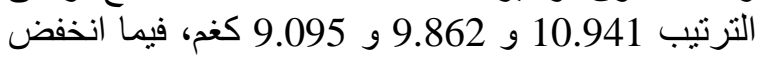
حاصل النبات الى اقل ما يمكن في النباتات المروية بات بالماء

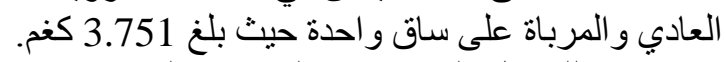

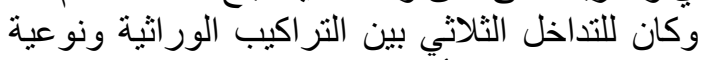

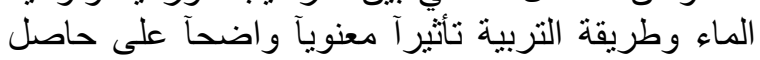

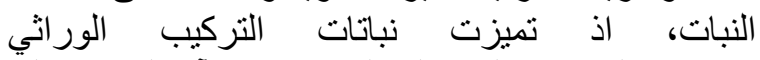

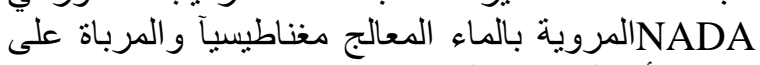

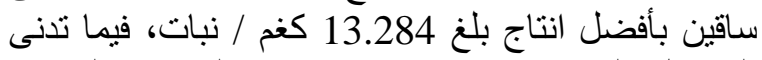

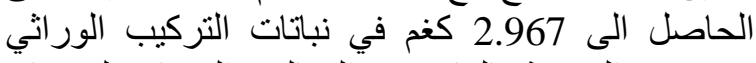
RAND المروية بالماء غير المعالج و المرباة على ساق التركي واحدة. 
جدول (4): تأثثر التركيب الوراثي والماء المعالج مغناطيسياً وطرائق التربية وتداخلاتها في متوسط حاصل النبات

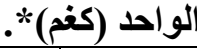

\begin{tabular}{|c|c|c|c|c|c|c|}
\hline \multirow{2}{*}{$\begin{array}{l}\text { التداخل } \\
\mathbf{V} \times \mathbf{W}\end{array}$} & \multicolumn{4}{|c|}{ طرائق التربية } & \multirow{2}{*}{ نوعية الماء } & \multirow{2}{*}{ الوراثية } \\
\hline & $\mathbf{T}_{3}$ & $\mathbf{T}_{2}$ & $T_{1}$ & $\mathbf{T}_{0}$ & & \\
\hline $\begin{array}{c}4.563 \\
b\end{array}$ & 6.465 & $\begin{array}{c}5.513 \\
\text { def }\end{array}$ & $\begin{array}{c}2.967 \\
f\end{array}$ & $\begin{array}{c}3.308 \\
f\end{array}$ & $\mathrm{~W}_{0}$ & \multirow[b]{2}{*}{$\mathbf{V}_{1}$} \\
\hline $\begin{array}{c}9.173 \\
\mathrm{a} \\
\end{array}$ & $\begin{array}{l}9.977 \\
a-d\end{array}$ & $\begin{array}{c}6.625 \\
\text { c-f }\end{array}$ & $\begin{array}{c}7.670 \\
b \text { - f }\end{array}$ & $\begin{array}{c}12.422 \\
\text { ab }\end{array}$ & $\mathbf{W}_{1}$ & \\
\hline $\begin{array}{c}4.142 \\
b\end{array}$ & $\begin{array}{c}3.315 \\
f\end{array}$ & $\begin{array}{c}3.975 \\
\text { f }\end{array}$ & $\begin{array}{c}4.192 \\
\text { ef }\end{array}$ & $\begin{array}{c}5.087 \\
\text { def }\end{array}$ & $\mathbf{W}_{\mathbf{0}}$ & \multirow{2}{*}{$\mathbf{V}_{2}$} \\
\hline $\begin{array}{c}8.898 \\
\text { a }\end{array}$ & $\begin{array}{c}8.955 \\
a-e\end{array}$ & $\begin{array}{c}13.284 \\
\text { a }\end{array}$ & $\begin{array}{c}6.377 \\
\mathrm{c}-\mathrm{f}\end{array}$ & $\begin{array}{c}6.977 \\
\mathrm{c}-\mathrm{f}\end{array}$ & $\mathbf{W}_{1}$ & \\
\hline $\begin{array}{c}4.789 \\
b\end{array}$ & $\begin{array}{c}5.056 \\
\text { def }\end{array}$ & $\begin{array}{c}5.930 \\
\text { c-f }\end{array}$ & $\begin{array}{c}4.094 \\
\text { ef }\end{array}$ & $\begin{array}{c}4.075 \\
\text { ef }\end{array}$ & $\mathbf{W}_{\mathbf{0}}$ & \multirow{2}{*}{$\mathbf{V}_{3}$} \\
\hline $\begin{array}{c}9.570 \\
\text { a }\end{array}$ & $\begin{array}{c}10.653 \\
\text { abc }\end{array}$ & $\begin{array}{c}12.913 \\
\text { a }\end{array}$ & $\begin{array}{c}6.828 \\
c \text { - f }\end{array}$ & $\begin{array}{c}7.887 \\
\text { b - f }\end{array}$ & $\mathbf{W}_{1}$ & \\
\hline & $\begin{array}{c}7.403 \\
\mathrm{~A} \\
\end{array}$ & $\begin{array}{c}8.040 \\
\text { A }\end{array}$ & $\begin{array}{c}5.354 \\
\text { B }\end{array}$ & $\begin{array}{c}6.625 \\
\text { AB } \\
\end{array}$ & \multicolumn{2}{|c|}{ متوسطات طرائق التربية } \\
\hline \multicolumn{7}{|c|}{ التداخل الثنائي بين التراكيب الوراثية وطرائق التربية } \\
\hline 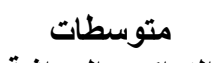 & \multicolumn{4}{|c|}{ طرائق التربية } & \multirow{2}{*}{\multicolumn{2}{|c|}{ التراكيب الوراثية }} \\
\hline الصرايليب أورابية & $\mathbf{T}_{3}$ & $\mathbf{T}_{2}$ & $T_{1}$ & $\mathbf{T}_{0}$ & & \\
\hline $\begin{array}{c}6.868 \\
A\end{array}$ & $\begin{array}{c}8.065 \\
\text { bc }\end{array}$ & $\begin{array}{c}8.504 \\
\text { abc }\end{array}$ & $\begin{array}{c}7.392 \\
\text { cd }\end{array}$ & $\begin{array}{c}10.143 \\
\text { a }\end{array}$ & \multicolumn{2}{|c|}{$\mathbf{V}_{1}$} \\
\hline $\begin{array}{c}6.520 \\
\mathrm{~A}\end{array}$ & $\begin{array}{c}9.713 \\
\text { ab }\end{array}$ & $\begin{array}{c}9.802 \\
\text { ab }\end{array}$ & $\begin{array}{c}10.085 \\
\text { a }\end{array}$ & $\begin{array}{c}9.051 \\
\text { abc }\end{array}$ & \multicolumn{2}{|c|}{$\mathbf{V}_{2}$} \\
\hline $\begin{array}{c}7.179 \\
\text { A }\end{array}$ & $\begin{array}{c}5.401 \\
\mathrm{e}\end{array}$ & $\begin{array}{c}5.785 \\
\text { de }\end{array}$ & $\begin{array}{c}5.326 \\
\mathrm{e}\end{array}$ & $\begin{array}{c}6.170 \\
\text { de }\end{array}$ & \multicolumn{2}{|c|}{$\mathbf{V}_{3}$} \\
\hline \multicolumn{7}{|c|}{ التداخل الثنائي بين نوعية الماء وطرائق التربية } \\
\hline متوسطات نوعية & \multicolumn{4}{|c|}{ طرائق التربية } & \multirow{2}{*}{\multicolumn{2}{|c|}{ نوعية الماء }} \\
\hline الماء & $\mathbf{T}_{3}$ & T2 & $\mathrm{T}_{1}$ & $\mathbf{T}_{0}$ & & \\
\hline $\begin{array}{c}4.214 \\
\text { B }\end{array}$ & $\begin{array}{c}4.945 \\
\text { cd }\end{array}$ & $\begin{array}{c}5.139 \\
\text { cd }\end{array}$ & $\begin{array}{c}3.751 \\
\text { d }\end{array}$ & $\begin{array}{c}4.157 \\
d\end{array}$ & \multicolumn{2}{|c|}{$\mathbf{W}_{\mathbf{0}}$} \\
\hline $\begin{array}{c}9.214 \\
\mathrm{~A}\end{array}$ & $\begin{array}{c}9.862 \\
a\end{array}$ & $\begin{array}{c}10.941 \\
\text { a }\end{array}$ & $\begin{array}{c}6.958 \\
\text { bc }\end{array}$ & $\begin{array}{c}9.095 \\
\text { ab }\end{array}$ & \multicolumn{2}{|c|}{$\mathbf{W}_{1}$} \\
\hline
\end{tabular}


جدول (5): تأثير التركيب الوراثي والماء المعالج مغناطيسياً وطرائق التربية وتداخلاتها في متوسط الحاصل الكلي

\begin{tabular}{|c|c|c|c|c|c|c|}
\hline \multirow{2}{*}{$\begin{array}{l}\text { التداخل } \\
\mathbf{V} \times \mathbf{W}\end{array}$} & \multicolumn{4}{|c|}{ طرائق التربية } & \multirow{2}{*}{ نوعية الماء } & \multirow{2}{*}{ الور الثية } \\
\hline & $\mathbf{T}_{3}$ & \begin{tabular}{l|l}
$\mathbf{T}_{2}$ \\
\end{tabular} & $\mathbf{T}_{1}$ & $\mathbf{T}_{\mathbf{0}}$ & & \\
\hline $\begin{array}{c}5.111 \\
b\end{array}$ & $\begin{array}{c}7.241 \\
c-f\end{array}$ & $\begin{array}{c}6.175 \\
\text { def }\end{array}$ & $\begin{array}{c}3.322 \\
f\end{array}$ & $\begin{array}{c}3.705 \\
\text { f }\end{array}$ & $\mathbf{W}_{\mathbf{0}}$ & \multirow{2}{*}{$\mathbf{V}_{1}$} \\
\hline $\begin{array}{c}10.274 \\
\text { a }\end{array}$ & $\begin{array}{c}11.174 \\
a-d\end{array}$ & $\begin{array}{c}7.420 \\
c-f\end{array}$ & $\begin{array}{c}8.590 \\
\text { b - f }\end{array}$ & $\begin{array}{c}13.912 \\
\text { ab }\end{array}$ & $\mathbf{W}_{1}$ & \\
\hline $\begin{array}{c}4.639 \\
b\end{array}$ & $\begin{array}{c}3.713 \\
\text { f }\end{array}$ & $\begin{array}{c}4.451 \\
\text { ef }\end{array}$ & $\begin{array}{c}4.694 \\
\text { ef }\end{array}$ & $\begin{array}{c}5.697 \\
\text { def }\end{array}$ & $\mathbf{W}_{\mathbf{0}}$ & \multirow{2}{*}{$\mathbf{V}_{2}$} \\
\hline $\begin{array}{c}9.966 \\
a\end{array}$ & $\begin{array}{c}10.029 \\
\mathrm{a}-\mathrm{e}\end{array}$ & $\begin{array}{c}14.878 \\
\text { a }\end{array}$ & $\begin{array}{l}7.142 \\
c-f\end{array}$ & $\begin{array}{l}7.813 \\
c-f\end{array}$ & $\mathbf{W}_{1}$ & \\
\hline $\begin{array}{c}5.363 \\
b\end{array}$ & $\begin{array}{c}5.662 \\
\text { def }\end{array}$ & $\begin{array}{c}6.641 \\
c-f\end{array}$ & $\begin{array}{c}4.585 \\
\text { ef }\end{array}$ & $\begin{array}{c}4.564 \\
\text { ef }\end{array}$ & $\mathbf{W}_{\mathbf{0}}$ & \multirow{2}{*}{$\mathbf{V}_{3}$} \\
\hline $\begin{array}{c}10.719 \\
a\end{array}$ & $\begin{array}{c}11.931 \\
\text { abc }\end{array}$ & $\begin{array}{c}14.463 \\
\text { a }\end{array}$ & $\begin{array}{c}7.648 \\
c-f\end{array}$ & $\begin{array}{c}8.832 \\
b-f\end{array}$ & $\mathbf{W}_{1}$ & \\
\hline & $\begin{array}{c}8.291 \\
\text { A }\end{array}$ & $\begin{array}{c}9.004 \\
\text { A }\end{array}$ & $\begin{array}{c}5.997 \\
\text { B }\end{array}$ & $\begin{array}{c}7.420 \\
\text { AB } \\
\end{array}$ & \multicolumn{2}{|c|}{ متوسطات طرائق التربية } \\
\hline \multicolumn{7}{|c|}{ التداخل الثنائي بين التراكيب الوراثية وطرائق التربية } \\
\hline متوسطات . & \multicolumn{4}{|c|}{ طرائق التربية } & \multirow{2}{*}{\multicolumn{2}{|c|}{ التراكيب الوراثية }} \\
\hline السراهيب الورابيه & $\mathbf{T}_{3}$ & $\mathbf{T}_{2}$ & $T_{1}$ & $\mathbf{T}_{0}$ & & \\
\hline $\begin{array}{c}7.692 \\
\text { A }\end{array}$ & $\begin{array}{c}9.207 \\
\text { ab }\end{array}$ & $\begin{array}{c}6.797 \\
\text { ab }\end{array}$ & $\begin{array}{c}5.956 \\
\text { b }\end{array}$ & $\begin{array}{c}8.809 \\
\text { ab }\end{array}$ & \multicolumn{2}{|c|}{$\mathbf{V}_{1}$} \\
\hline $\begin{array}{c}7.302 \\
\mathrm{~A}\end{array}$ & $\begin{array}{c}6.871 \\
\text { ab }\end{array}$ & $\begin{array}{c}9.665 \\
\text { ab }\end{array}$ & $\begin{array}{c}5.918 \\
\text { b }\end{array}$ & $\begin{array}{c}6.755 \\
\text { ab }\end{array}$ & \multicolumn{2}{|c|}{$\mathbf{V}_{2}$} \\
\hline $\begin{array}{c}8.040 \\
\text { A } \\
\end{array}$ & $\begin{array}{c}8.797 \\
\text { ab }\end{array}$ & $\begin{array}{c}10.552 \\
\text { a }\end{array}$ & $\begin{array}{c}6.117 \\
\text { b }\end{array}$ & $\begin{array}{c}6.698 \\
\text { ab } \\
\end{array}$ & \multicolumn{2}{|c|}{$\mathbf{V}_{3}$} \\
\hline \multicolumn{7}{|c|}{ التذاخل الثنائي بين نوعية الماء وطرائق التربية } \\
\hline متوسطات نوعية & \multicolumn{4}{|c|}{ طرائق التربية } & \multirow{2}{*}{\multicolumn{2}{|c|}{ نوعية الماء }} \\
\hline الماء & T3 & $\mathbf{T 2}$ & T1 & T0 & & \\
\hline $\begin{array}{c}5.037 \\
\text { B }\end{array}$ & $\begin{array}{c}5.538 \\
\text { cd }\end{array}$ & $\begin{array}{c}5.756 \\
\text { cd }\end{array}$ & $\begin{array}{c}4.201 \\
d\end{array}$ & $\begin{array}{c}4.655 \\
d\end{array}$ & \multicolumn{2}{|c|}{$\mathbf{W}_{\mathbf{0}}$} \\
\hline $\begin{array}{c}10.319 \\
\text { A }\end{array}$ & $\begin{array}{c}11.045 \\
\text { a }\end{array}$ & $\begin{array}{c}12.253 \\
\text { a }\end{array}$ & $\begin{array}{c}7.793 \\
\text { bc }\end{array}$ & $\begin{array}{c}10.186 \\
\text { ab }\end{array}$ & \multicolumn{2}{|c|}{$\mathrm{W}_{1}$} \\
\hline
\end{tabular}

$$
\text { للبيت البلاستيكي (طن)*. }
$$

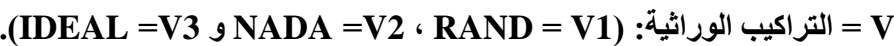

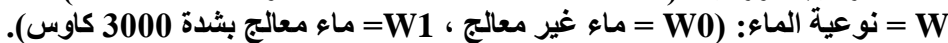
T = طرائق التربية: (T0= بدون تقليم ، T1= التربية على سلق واحدة ، T2= التربية على ساقين و T3= التربية على ثلاثة سيقان). 


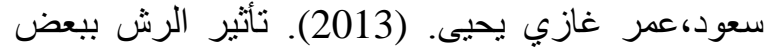

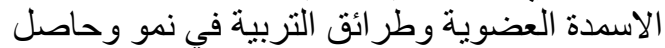

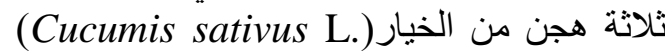
في البيوت المحمية.رسالة ماجستير.قسم البرازية البستنة وهندسة الحدائق. كلية الزراعة. جامعة دئة ديالى. العراق.

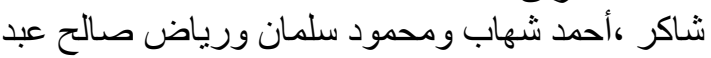

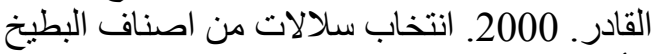

الأصفر المحلي. مجلة الزراعة العر اقية. 5 (7) (7) : $.58-46$ الصحاف ، فاضل حسين. (1995). تأثير عدد السيقان و التغذية الورقية في الحاصل ومكوناته في تئي

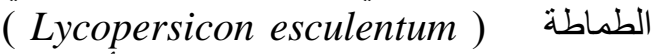
تحت ظروف البيوت البلاستيكية المدفأة. مجلة العلوم الزراعية العر اقية ـ 26 العدد 2 : 59 -

العبدلي، معاذ محيي محمد شريف. (2007). تحسين Cucumis melo بعض صفات البطيخ الأصفر

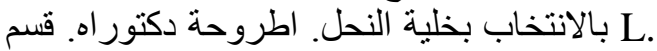

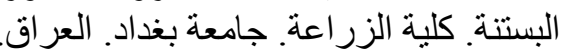
محمد، عبد العظيم كاظم. (1977). مبادئ تغذية الزية النبات.

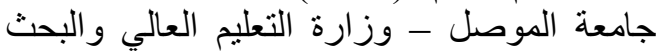

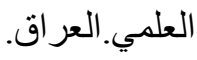

مطلوب، عدنان ناصر وعز الدين سلطان محمد و كريم

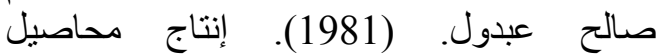

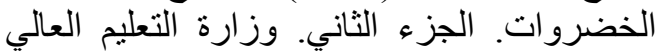

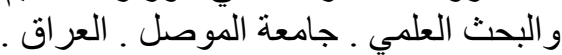

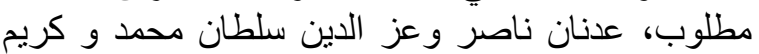

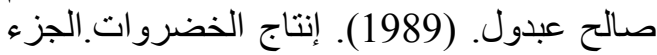
الثاني. وزارة التعليم العالي والبحث البحث العلمي .

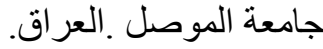
المعاضيدي، علي فاروق ومصطفى رشيد العرايد القيسي و أديب

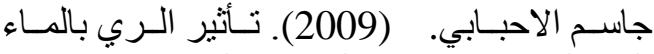

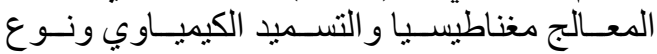

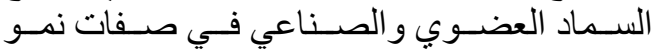

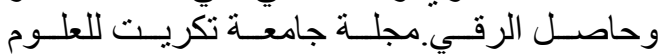
الزر اعية. 9 (3): 190-183.

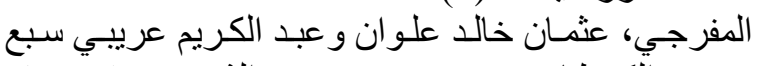

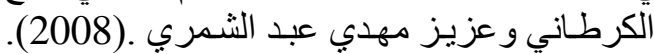

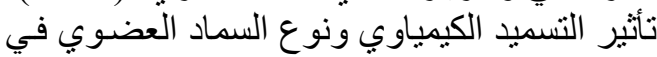

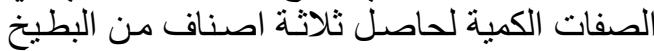

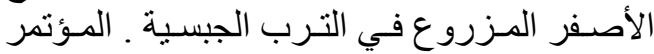

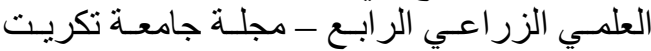
(العدد الخاص بالمؤتمر ).الصفحات: 269 - 280.

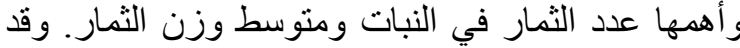

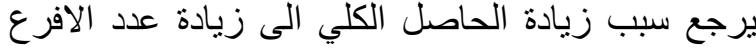

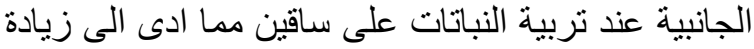

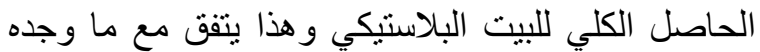

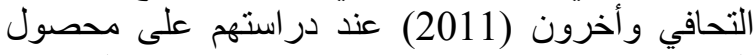

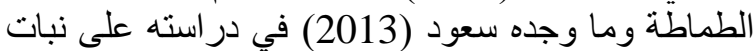

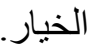

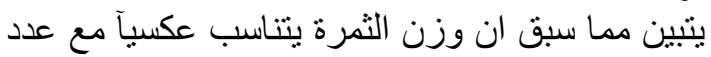

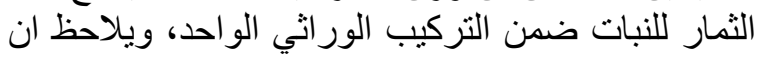

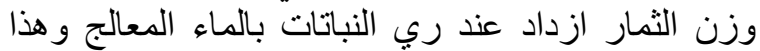

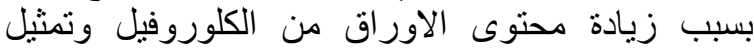

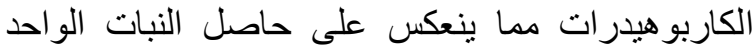

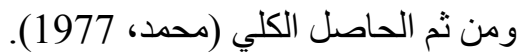

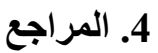

التحافي، سامي علي عبد المجيد وموسى محمد حمزة و ولئر

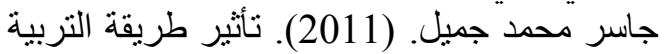
و الرش بالميكرونيت 15 في نمو وحاصل الطماطية صنف نيوتن في البيت البلاستيكي. مجلة الفرونة الفرات للعلوم الزر اعية. 3 (4): 91 - 91 - 99.

الجهاز المركزي المعلومات.(2013). وزارة التخطيط. جمهورية التوبية

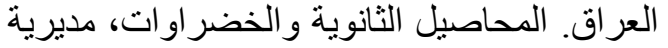
الإحصاء الزراعي، وزارة التئية التخطيط والتعاون

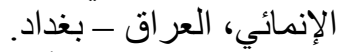

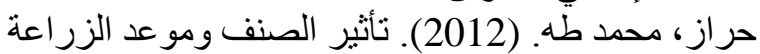

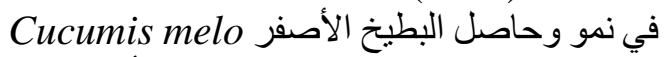
Lي البيوت البلاستيكية غير المدفأة، رسالة

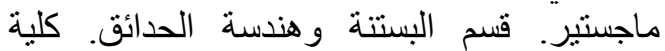

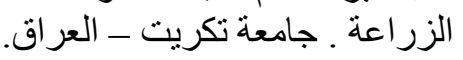

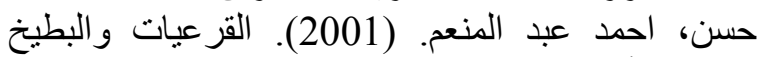

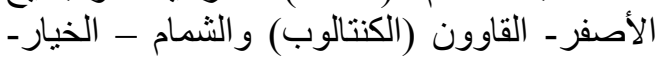

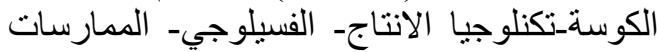

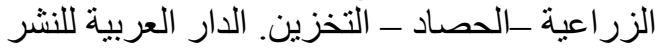
و التوزيع - القاهرة. جمهورية مصر العزية العربية. الحمداني، شامل يونس حسن.(2013 ). التباين الورة التئي

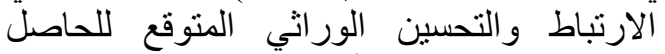

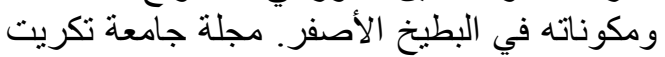
للعلوم الزر أعية ـ 13 (2): 236 - 227. الراوي، خاشع محمود و عبد العزيز خلف الله اله. (223) (2000).

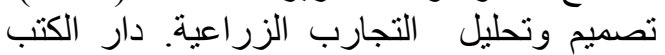
للطباعة والنشر - جامعة الموصل. الطبعة الثانية. 488 صفحة. 


\section{REFERENCES}

Basant L. and . M. S. Grewal. (2009). Magnetic treatment of irrigation water: Its effects on vegetable crop yield and water productivity . Agric. water management 96:1229 - 1236.

Hilal M. H. and Hilal M. M. (2000). Application of magnetic technologies in desert agriculture II - Effect of magnetic treatments of irrigation water on salt distribution in olive and citrus fields and induced changes of ionic balance in soil and plant. Egypt. J. Soil. Sci., 40 (3): 423 435.

Kronenberg K. J (1993) Magnetized: More alluring facts about treating water with magnets. Aqua Magazine, Sept, 20-23.

Long R.L. (2005). Improving fruit soluble solids content in melon reticuiates group in the Australian production system. A dissertation submitted to plant sciences group. Primary industries research center. School of biological and environmental science. Fac. of Arts Health and Sci. Center Queens Land University, Rokhampton, Australia, PP. 362.
Petkove M. (1994). The technology of growing determination tomato cultivars Gardeners FAO,15 (1) 14:17 C.F Horti Abst .Vo1. 44, Abst. 3290.

SAS (2001). Statistical Analysis System. SAS Institute Inc. Cary. N. C.

Shoemaker H. and William E. (2002). Estern Muskmelon cultivar observation Univ. of Illinois, U.S.A.

Smith (2005). Magnetic water hydromag. The water charger. www.healthwalk.com.

Takeshi K., Akira A., Katsumi S. and Takayuki S. (2006). The effects of nighttime temperature, shading and branch training on the bearing and yield of parthenocarpic eggplant in forcing culture. Bulletin of Fukuoks Agric. Res. Center, Japan, 25: 33-36.

Yang B., Yonghong G., Chunling W. and Xuewen L. (2007). Melon production in China. Acta Hort. (ISHS) 731:493-500. 